\title{
A PRACH Preamble Generation and Detection Model for 5GNR Systems
}

\author{
Puneeth $\mathrm{K} \mathrm{M}^{*_{1}}$, Poornima M S2, \\ ${ }^{*}$ Assistant Professor, Department of ECE, SJCE,JSS-STU, Mysuru, Karnataka, India \\ ${ }^{2} \mathrm{PG}$ Scholar, MTech( NIE), Department of ECE, SJCE, JSS-STU, Mysuru, Karnataka, India
}

\begin{abstract}
Article Info

Volume 8, Issue 4

Page Number: 98-105

Publication Issue :

July-August-2021

\section{Article History}

Accepted : 06 July 2021

Published: 13 July2021

The basic idea of $5^{\text {th }}$ generation New Radio (5GNR) is to have very high data rate and to make it work efficiently for all Internet of Things (IOT) applications like healthcare, Automotive, Industrial etc. applications. This paper provides the Orthogonal Frequency Division Multiple Access (OFDM) baseband signal generation and detection method for Physical Random-Access Channel (PRACH). The proposed model provides four scenarios of preamble detection i.e., Preamble detection probability, Miss-detection probability, False alarm probability and null. We achieved the target of $99 \%$ of Probability of Detection and less than $0.1 \%$ of False-alarm probability at certain SNR as specified according to 3gpp standard requirements when tested in Additive White Gaussian Noise (AWGN) channel and Extended Typical Urban (ETU) channel.

Keywords: PRACH, Preamble, Detection Probability, Miss-detection probability, False alarm.
\end{abstract}

\section{INTRODUCTION}

The 5GNR technology uses various new technologies to reach its target to provide a high data rate. 5GNR technology has some target scenarios i.e., the enhanced Mobile Broadband (eMBB), the UltraReliable Low Latency Communication (URLLC), and the massive Machine Type Communication (eMTC). Preamble detection is the first step for User Equipment (UE) to obtain initial access by (Random Access Channel) RACH Procedure. UE performs the RACH procedure to obtain the uplink synchronization with gNB. The Random Access (RA) procedure is initiated by UE by transmitting RA preambles which are selected randomly from the pool of available preambles on the Physical random access channel (PRACH). 5G-NR has 64 preambles in each time-frequency PRACH occasion which are the same as LTE [1].

Several studies, surveys, and works have been done and published on preamble detection. To increase the probability of detection of preamble from the preamble receiver various methodologies are proposed which results in a detection probability of $99 \%$. 
In the paper, an approach on preamble generation in 5G NR multi-Subcarrier spacings (SCS) and also the random access methods supported multi-beam capabilities of UE and gNodeB (gNB) are implemented. The presented preamble generation method can reduce the influence occur due to intercarrier interference, also the Random Access (RA) procedure provides the opportunity of beam-based approach in addition to increase the probability of access upon conventional power domain procedure[7].

Before starting with the implementation of the new model, it is important to understand the root causes for preamble miss detection at the receiver in the conventional method. According to 3gpp standard [6] (38.141-1 section 8.4.1.5) at some specific SNR values and for the set of PRACH configurations and propagation conditions, the probability of PRACH detection should be greater than or equal to $99 \%$ and miss detection probability less than $0.1 \%$ and it is also mentioned that the receiver model should have the ability to detect the preamble under any static conditions and multipath fading propagation conditions for given SNR.

Preamble miss detection can occur due to the following error cases as specified in 3gpp specifications.

- Incorrect Preamble detection

- No preamble detection

- Proper preamble detection but with wrong Timing Estimation.

The objective of this paper is to address the first (false detection) and second (miss-detection) error case i.e., No Preamble detection in physical random access channel. This paper describes the root cause for gNB for not detecting the preamble sent by UE. In conventional method if $\mathrm{gNB}$ doesn $\square \mathrm{t}$ detect the preamble sent by UE, it will not send the random
Access Response (RAR) back to UE. UE will wait for certain period and then resends the preamble by performing power ramping.

Further sections of this paper are as follows: section II of the paper describes the existing system model for preamble detection with its limitations. Section III describes the RACH Preamble generation, Preamble Transmission and Preamble Detection. Section IV provides the Methodology, Section $\mathrm{V}$ provides the simulated results and analysis. And the final section VI and VII gives the conclusion and future work of this paper respectively.

\section{RELATED WORK}

Various study has been done to detect the preamble with accuracy as specified in standards and to reduce the miss detection of preamble from past LTE through 5G technology. The studies are done through different direction to achieve the same motive. The survey on same work includes common approaches like Several Preamble detection algorithms are proposed[3], new PRACH receiver design, power ramping, beam switching[4], usage of different channel i.e., AWGN, ETU etc., preamble detection for frequency domain samples[8] for WLAN systems. Preamble detection is also implemented for various applications like high speed railways, tunnel coverage etc.

Eun-Sook Jin et al [1] proposed a method of detection of preamble in frequency domain and introduced a concept of sample based FFT. They presented the threshold determination criteria through power ratio signal analysis and verification is done using simulation. They proposed system provides an approach which allows sample based operation in frequency domain and presented the improvement in performance when compared to existing preamble detection system in terms of SNR, but they have also mentioned that the proposed system is unsafe to the impact of carrier frequency aggregation (CFO). 
Tuan Anh Pham et al [3], proposed a algorithm for preamble detection for 5G PRACH systems with multi-detection steps to reduce the detection of false peaks due to various conditions like noise, multipath fading, doppler shift, timing offset and frequency offset. They introduced a new seven steps sub-module which detects the preamble presence in Power Delay Profile (PDP) from matching filter. The proposed method achieves the targets of detection and False alarm probability with condensed detection threshold under SNR values.

Gweon-Do Jo et al [2] proposed a paper by evaluating the performance of physical random access channel for 5G systems. They considered AWGN channel and ETU channel with value for simulation configured as, $61.44 \mathrm{Mbps}$ of sampling rate, subcarrier spacing (SCS) of $30 \mathrm{KHz}$ with $3.7 \mathrm{GHz}$ center frequency and system bandwidth of $40 \mathrm{MHz}$. The target of 3gpp standard release 15 is achieved for preamble miss-detection probability.

\section{PRACH IN 5G SYSTEMS}

\section{A. RACH Preamble}

The RA preamble can be built by concatenating several short sequences, each sequence will have the identical length as an OFDM symbol for other NR UL signals. These short sequences may be processed with identical FFT sizes. This preamble format also helps in enabling fast time varying channels, enormous frequency offsets, phase noise etc.

The RA preamble has unique signal format which includes three main components, i.e., Preamble sequence, Cyclic Prefix, Guard time. Which can be represented as $T_{s e q}, T_{C P}$ and $T_{G T}$ respectively. General preamble format generation is shown in Figure 1 and Preamble Format.

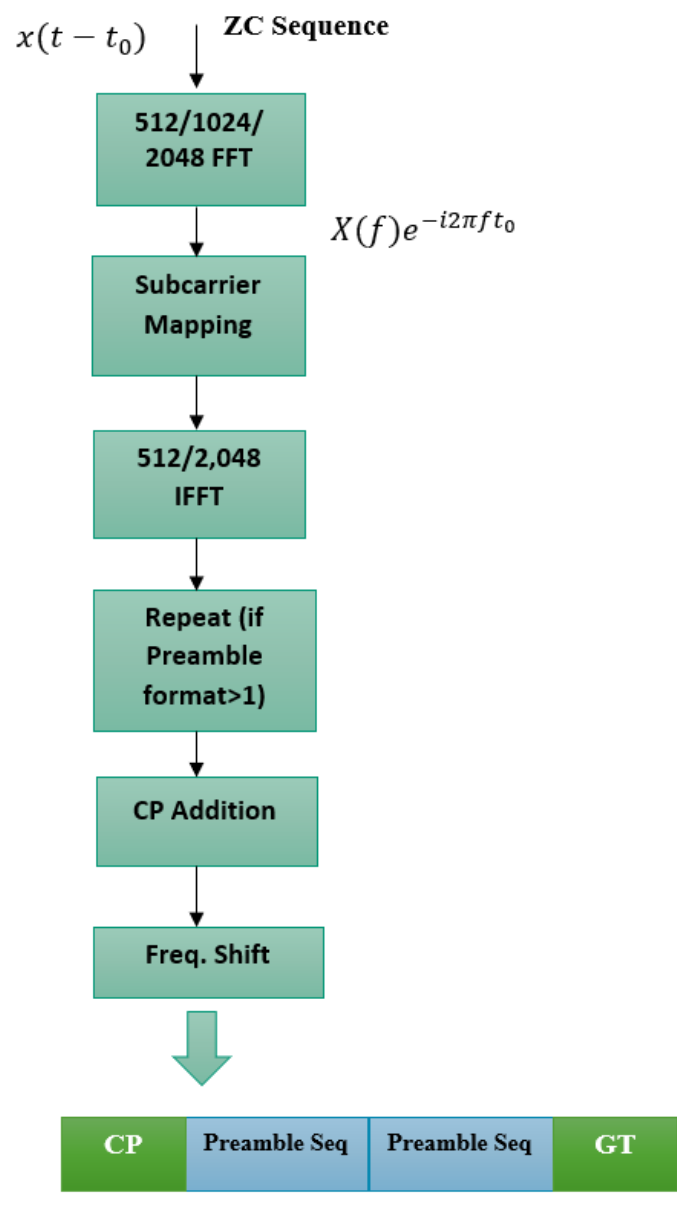

Figure 1: Preamble Sequence Generation

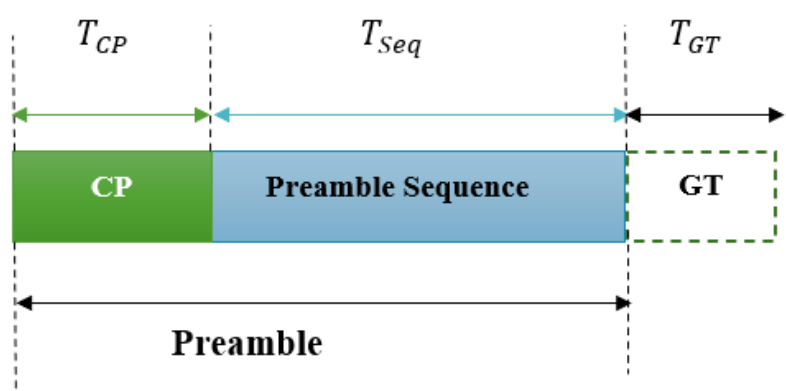

Figure 2: Preamble Format

Preamble sequence is nothing but a $\mathrm{ZC}$ sequence generated uniquely for every UE. As specified in 3gpp specifications, The set of preamble sequences $x_{u, v}(n)$

$$
\begin{aligned}
& x_{u, v}(n)=x_{u}\left(\left(n+C_{v}\right) \bmod L_{R A}\right. \\
& x_{u}(i)=e^{-j \frac{j u(i+1)}{L_{R A}}, i=0,1, \ldots, L_{R A}-1}
\end{aligned}
$$


Where $u$ represents the preamble root sequence index and the length of RA preamble sequence is represented by $L_{R A}$. The value of $L_{R A}$ can be 139,839 , 571 or 1151 depending on the preamble format configuration given in Tables 6.3.3.1-1 and 6.3.3.1-2 in 3gpp TS 38.211[5]. $C_{v}$ is the cyclic shift of each preamble root sequence index.

The same expression can be expressed in frequency domain as,

$$
y_{u, v}(n)=\sum_{m=0}^{L_{R A}} x_{u, v} \cdot e^{-\frac{2 \pi m \pi}{L_{R A}}}
$$

Like LTE, 5GNR also uses different types of preamble formats which are grouped into two categories, i.e., Long Formats and Short Formats and classified based on SCS used in the preamble. This classification is made to provide cell size coverage and time delay, and to provide flexibility regarding capacity. According to 3gpp standard specified in 38.211[5], if the PRACH Preamble is using the SCS of $1.25 \mathrm{KHz}$ or $5 \mathrm{KHz}$, then the Long preamble formats, Format $0 / 1 / 2 / 3$ can be used with ZC length 839 . Long preamble formats are more likely used for macro deployments or large cells. Recent 3gpp release has included two more ZC lengths i.e., 571 and 1151.

If the PRACH Preamble is using the SCS of $15 \mathrm{KHz}$, $30 \mathrm{KHz}, 60 \mathrm{KHz}$ or $120 \mathrm{KHz}$, then preamble format can be from Short sequence group with $\mathrm{ZC}$ length of 139[4]. Short sequence preamble formats are dedicated for small cells and indoor deployments.

\section{B. Preamble Transmission}

In $5 \mathrm{G} \mathrm{NR}$, the network controls the timing of uplink transmission based on time adjustments commands (closed loop timing control) which are provided regularly. Before UE sends preamble, closed-loop timing control will not be there, it is uncertain in the preamble reception. Larger cells could have preamble reception timing of $100 \mu$ s or more. In general, gNB scheduler will ensures there should not be any other transmissions in uplink resourses where preamble transmission can take place.

\section{PRACH Detection}

The main objective of PRACH detector is detection of transmitted preamble from the PRACH transmitter through RA channel at proper cyclically shifted root sequence and also to know the propagation delay or timing offset of UE which transmits that preamble. A typical PRACH receiver model is presented in Figure 3. After time domain signal is received, cyclic prefix will be removed as a first step and to have the samples in Frequency Domain(FD), number of FFT $\left(N_{F F T}\right)$ point Fast Fourier Transform (FFT) is taken. The obtained samples in FD are correlated with the root sequence as next step i.e., complex multiplication of received FD samples with root sequences. As UE might have used preamble indices for transmission, according to some hypothesis, for detection process at $\mathrm{gNB}$, ranging from the starting root sequence up to configured number of root sequences will be used.

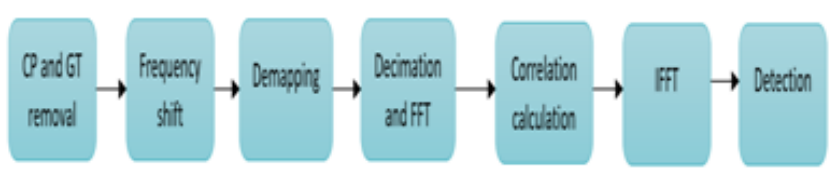

Figure 3: Block diagram of PRACH detector

After analyzing energy in time using Inverse Fast Fourier Transform (IFFT), to detect the correlation peaks and to detect the location within the window, cross correlation is performed. Before peak detection, it is possible to process the received preamble independently, also possible to combine time domain energies using non-coherent PRACH detector, for each of the received symbols and antennas. But, the complexity and performance of the non-coherent detector sometimes not suitable with PRACH formats with high repetitions must operate in low SNR values as expected. 


\section{METHODOLOGY}

When UE sends the preamble to gNB, gNB can detect the preamble if the signal is proper. The preambles with low SNR value are more prone for miss detection as that signal is already degraded by noise and it is difficult to find the peaks to perform Auto/Cross correlation at the receiver end. We propose a model at $\mathrm{gNB}$ side to enhance preamble detection and decrease false alarm probability, we mainly focused on four different conditions listed below,

1. Probability of Detection ( $\left.P_{\text {detection }}\right)$

2. Probability of Miss-detection

$$
\text { ( } P_{\text {miss-detection }} \text { ) }
$$

3. Probability of False-Alarm (

$P_{\text {false-alarm })}$

4. Invalid $\left(P_{\text {mull }}\right)$

$P_{\text {detection }}$ represents the probability of total number of preambles detected out of all the received samples, i.e., UE sends the preamble and gNB detects the preamble successfully. $\mathrm{P}$ miss-detection represents the probability total number of preambles not detected in the received samples i.e., UE sends the preamble gNB doesn $\square t$ detect the preamble. $\mathrm{P}$ false-alarm represents the probability of number of false preambles detected at gNB. False preambles are those which are sent or not sent from UE but detected at gNB by detecting the high noise peaks which falls above the threshold and considering the noise peaks as preamble. $\mathrm{P}$ invalid represents the condition where no preambles sent from UE and no preambles detected at gNB.

The below Figure 5 shows the sequence of processes performed at PRACH receiver. To reduce the probability of miss detection we have performed a process a nullifying the unused cyclic shifts at the receiver. The number of cyclic shifts $\left(\mathrm{N}_{\mathrm{cs}}\right)$ need to be performed depends on the ( $\left.\mathrm{N}_{\mathrm{cs}}\right)$ value. After the root $\mathrm{ZC}$ sequence is generated it is shifted cyclically based on $\left(\mathrm{N}_{\mathrm{cs}}\right)$ values to generate all the 64 preambles. The number of root sequence required to generate 64 preambles is dependent on cell size. For a cells with smaller size (about $1.5 \mathrm{~km}$ ) only one root sequence is enough to generate all 64 preambles. But for the cells with larger size, more than one root sequence is required. The result of correlation of cyclically shifted sequences from same root sequences is zero and with different root sequences is non-zero. So it is good to have less root sequences. This can be achieved by removing the cyclically shifted sequences which are not used in the pool of preambles.

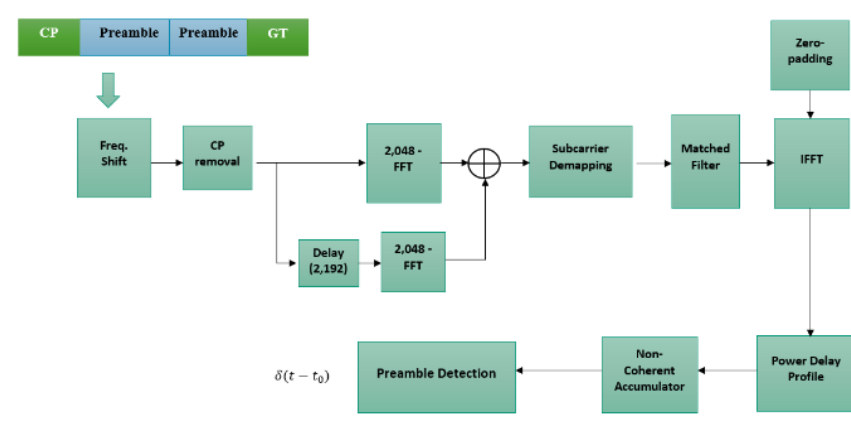

Figure 5: Preamble Detection

The received signal in time domain is sent FFT module to convert it into frequency domain with SCS specified in simulation configuration, then subcarrier de-mapping is performed to select the subcarriers containing the RA preamble. The sequence is then correlated with root sequence in frequency domain and IFFT is performed along with Zero-padding. Next step is to compute the power delay profile and noncoherent accumulation among the sequences and the antennas and finally Preamble sequence detection with detection algorithm.

To identify the correct preamble and to calculate the probability of detection, we will check the preamble index of the received preamble and compared with configured values, if it matches then that preamble is considered as correct preamble. If the preamble index does not matches with the configured value then that preamble is considered as false-alarm. 


\section{RESULTS AND ANALYSIS}

The results of simulation is presented in this section. The performance of the implemented model is evaluated in computer simulations with four conditions i.e., Probability of detection, Probability of miss-detection, Probability of False-alarm and null. The resultant graphs of the simulations shows that the implementation reached the target of 0.99 of Probability of detection and 0.1 of false alarm probability and miss detection probability at certain SNR. The simulation is performed in two noisy channels i.e., AWGN channel and ETU channel. Configuration parameters considered are mentioned in Table 1.

\begin{tabular}{|l|l|}
\hline Parameters Subcarrier & Specifications \\
\hline $\begin{array}{l}\text { Carrier subcarrier } \\
\text { spacing }\end{array}$ & $1.25 \mathrm{KHz}$ \\
\hline $\begin{array}{l}\text { PRACH } \\
\text { spacing }\end{array}$ & 13 \\
\hline NCS & 100 \\
\hline Number of sub-frames & 2 \\
\hline $\begin{array}{l}\text { No. of Transmit } \\
\text { Antenna } \quad \text { Receive }\end{array}$ & 2 \\
\hline $\begin{array}{l}\text { No. of } \\
\text { Antennas }\end{array}$ & $400 \mathrm{~Hz}$ \\
\hline Frequency offset & 0 \\
\hline Preamble Format &
\end{tabular}

Table 1: Simulation configurations

Figure 6 shows the simulations results obtained when Extended Typical Urban channel is considered. We have achieved false alarm probability of less than 0.1 at SNR -10db and miss detection probability of less than 0.1 at $-4 \mathrm{db}$

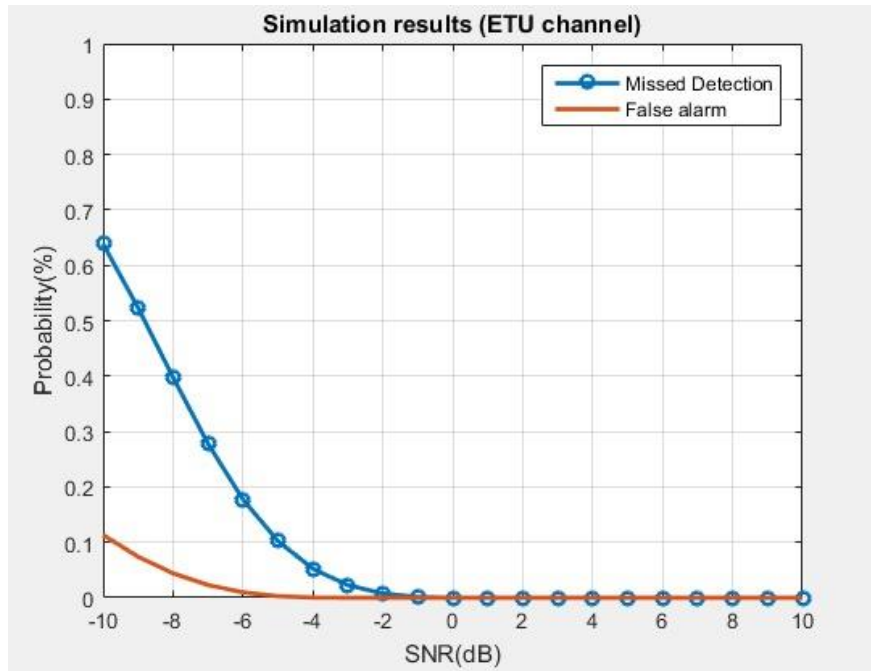

Figure 6: Simulation Results for ETU channel

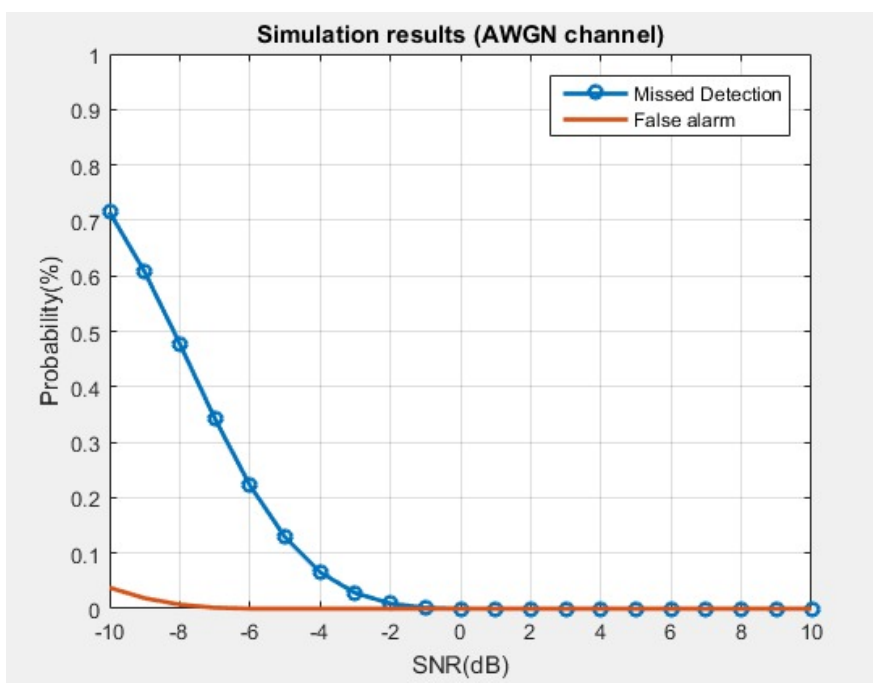

Figure 7: Simulation Results of AWGN channel

Figure 7 shows the simulation results obtained for probability of miss detection and false alarm probability in Additive White Gaussian Noise channel and we have achieved miss detection probability less than 0.1 at SNR $-4 \mathrm{db}$ and false alarm probability less than 0.1 at SNR less than -10db. By performing simulations in both AWGN and ETU channel, the results are concluded as shown in Table 2. 


\begin{tabular}{|l|l|}
\hline Channel & $\begin{array}{l}\text { Less than 0.1 probability } \\
\text { achieved at SNR in DB }\end{array}$ \\
\hline AWGN & $\begin{array}{l}\text { False Alarm: less than -10db } \\
\text { Miss Detection: }-4 \mathrm{db}\end{array}$ \\
\hline ETU & $\begin{array}{l}\text { False Alarm: }-10 \mathrm{db} \\
\text { Miss detection: }-4 \mathrm{db}\end{array}$ \\
\hline
\end{tabular}

Table 2: Simulation results

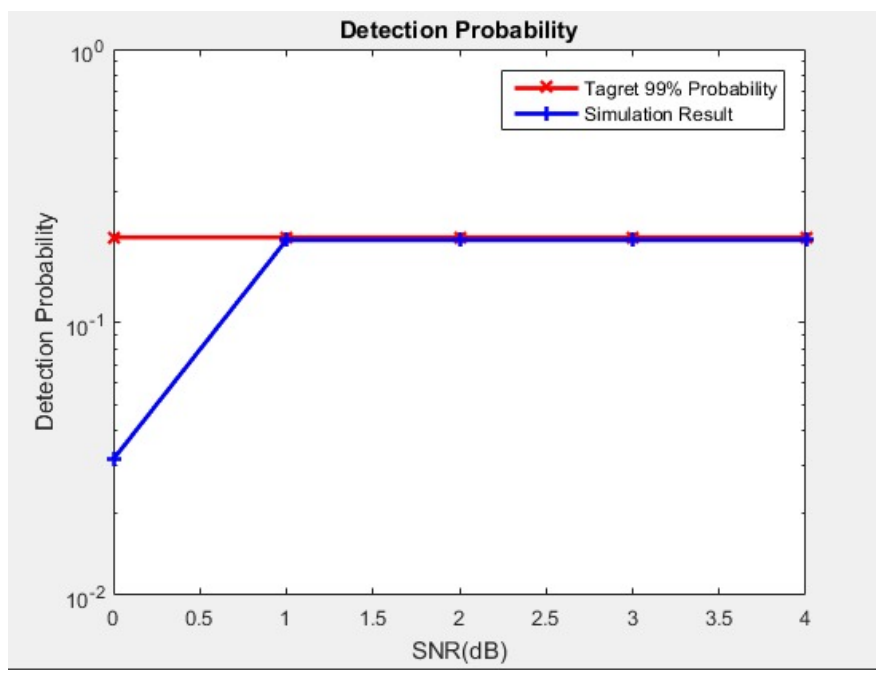

Figure 8: Simulation Results of detection probability

Figure 8 shows the simulation results of Probability of detection achieved target of $99 \%$. The target is achieved at SNR value of $1 \mathrm{db}$.

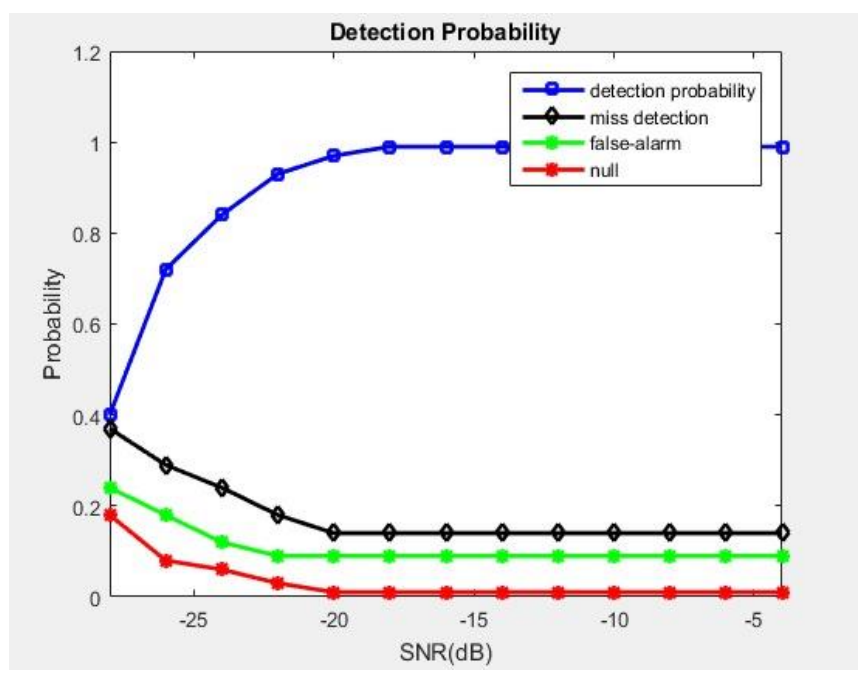

Figure 9: Simulation Results of all 4 conditions
Figure 9 shows the simulation results of all 4 conditions considered that is, Probability of detection, probability of miss detection, false alarm probability and Null. $P_{\text {detection }}$ is achieved at SNR $-15 \mathrm{db}$.

\section{CONCLUSION}

In this paper, Preamble sequence is generated, and baseband level simulation is performed to evaluate the performance of PRACH in 5G NR systems according to 3gpp standard. By using the new approach of nullifying the unused cyclic shifts of the root sequence, we can achieve the target of $99 \%$ of $P_{\text {detection }}$ at SNR $-15 \mathrm{db}$, less than $0.1 P_{\text {miss-detection at }}$ SNR $-4 \mathrm{db}$ and $P_{\text {false-alarm }}$ at $\mathrm{SNR}$ at $-10 \mathrm{db}$ and $P_{\text {mull }}$ value to 0 .

\section{FUTURE WORK}

The detection probability is mainly related to received signal strength and the threshold value set at the receiver. There is a possibility that noise with high peaks can be detected as a preamble. So it is necessary to predict the noise from the received signal and regenerate the estimated noise and subtracting it from received signal which reduces the SNR and increases the detection probability.

\section{REFERENCES}

[1] Eun-Sook Jin and Hyuncheol Park, DA Novel Frequency Domain Preamble Detection for OFDM based WLAN Systems $\square$ in 2020, IEEE.

[2] Gweon-Do Jo and Young Ha Lee, DPerformance Evaluation of Physical Random Access Channel in 5G New RadioD in 2019 International Conference on Advanced Communications Technology (ICACT).

[3] Tuan Anh Pham, Bang Thanh Le, DA proposed preamble detection algorithm for 5G-PRACHD in 2019 International Conference on Advanced Technologies for Communications (ATC). 
[4] I5G NR RACH Preamble types: Long and Short PreamblesQ, July 10, 2019, https://www.techplayon.com

[5] 3GPP TS 38.211 v15.8.0, "Physical channels and modulation," 12-2019.

[6] 3GPP TS 38.141-1 v15.0.0., April 2019

[7] Qi Xiong, Bin Yu, Chen Qian, Xiaojiao Li, Chengjun Sun, QRandom Access Preamble Generation and Procedure Design for 5G-NR SystemL in 2018.

\section{Cite this article as :}

Puneeth K M, Poornima M S, "A PRACH Preamble Generation and Detection Model for 5GNR Systems", International Journal of Scientific Research in Science, Engineering and Technology (IJSRSET), Online ISSN : 2394-4099, Print ISSN : 2395-1990, Volume 8 Issue 4, pp. 98-105, July-August 2021. Available at doi : https://doi.org/10.32628/IJSRSET218426 Journal URL : https://ijsrset.com/IJSRSET218426 